\title{
Short topotecan-based induction regimen in newly diagnosed high-risk neuroblastoma
}

\author{
Maria Antonietta De Ioris ${ }^{a, *}$, Aurora Castellano ${ }^{a}$, Ilaria Ilari ${ }^{a}$, Maria Carmen \\ Garganese $^{b}$, Gianluigi Natali ${ }^{b}$, Alessandro Inserra ${ }^{c}$, Rita De Vito ${ }^{d}$, Lucilla Ravà ${ }^{e}$, Maria \\ Debora De Pasquale ${ }^{a}$, Franco Locatelli ${ }^{a, f}$, Alberto Donfrancesco ${ }^{a}$, Alessandro Jenkner ${ }^{a}$ \\ a Department of Paediatric Oncology-Hematology, Ospedale Pediatrico Bambino Gesù IRCCS, Rome, Italy \\ ${ }^{\mathrm{b}}$ Imaging, Ospedale Pediatrico Bambino Gesù IRCCS, Rome, Italy \\ c Pediatric Surgery, Ospedale Pediatrico Bambino Gesù IRCCS, Rome, Italy \\ d Pathology Unit, Ospedale Pediatrico Bambino Gesù IRCCS, Rome, Italy \\ e Epidemiology Unit, Ospedale Pediatrico Bambino Gesù-IRCCS, Rome, Italy \\ ${ }^{\mathrm{f}}$ University of Pavia, Italy
}

\section{A R T I C L E I N F O}

Article history:

Received 25 July 2010

Received in revised form 25 October 2010

Accepted 27 October 2010

Available online 26 November 2010

Keywords:

Neuroblastoma

Metastasis

Children

Topotecan

Remission

Induction

\begin{abstract}
A B S T R A C T
Purpose: Topotecan is an active drug in relapsed neuroblastoma. We investigated the efficacy and toxicity of a topotecan-based induction regimen in newly diagnosed neuroblastoma.

Methods: Patients older than 1 year with either metastatic or localised stage 2-3 MYCNamplified neuroblastoma received 2 courses of high-dose topotecan (HD-TPT) $6 \mathrm{mg} / \mathrm{m}^{2}$ and high-dose cyclophosphamide (HD-CPM) $140 \mathrm{mg} / \mathrm{kg}$, followed by 2 courses of ifosfamide, carboplatin and etoposide (ICE) every 28 days. After surgery on primary tumour, a fifth course with vincristine, doxorubicin and CPM was given, followed by high-dose chemotherapy with stem cell support. Response was assessed in accordance with the International Neuroblastoma Response Criteria.

Results: Of 35 consecutive patients, 33 had metastatic disease. The median length of induction phase was 133 days (range 91-207) and time to high-dose chemotherapy was 208 days (range 156-285). The median tumour volume reduction was 55\% after two HD-TPT/HD-CPM courses and $80 \%$ after four courses. Radical surgery was performed in 16/27 patients after chemotherapy. After the fifth course, 29/34 patients (85\%) had achieved a partial remission (12) or a CR/very good partial remission (17). CR of metastases was achieved in 13/32 (41\%) and bone marrow was in complete remission in $16 / 24$ patients (67\%). Grade 4 neutropenia and/or thrombocytopenia occurred in 100\% of HD-TPT/HD-CPM and in 95\% of ICE courses, while non-haematological toxicities were manageable.

Conclusions: These data indicate that our induction regimen is feasible and well tolerated. A major response rate of $85 \%$ with $41 \%$ complete metastatic response confirms this regimen as effective induction in high-risk neuroblastoma.
\end{abstract}

(c) 2010 Elsevier Ltd. All rights reserved.

\footnotetext{
* Corresponding author: Tel./fax: +3906 68592574 .

E-mail address: deioris@opbg.net (M.A. De Ioris). 


\section{Introduction}

The improved characterisation of neuroblastoma biology has resulted in the possibility of adopting tailored treatment strategies. ${ }^{1}$ However, the outcome of patients with high-risk neuroblastoma remains unsatisfactory with a 3-year eventfree survival (EFS) of less than $40 \%$ despite intensive treatment. ${ }^{2-5}$

The goal of induction is to achieve a reduction of both primary and metastatic tumour burden. A correlation between dose intensity during induction and response/survival rate has been demonstrated. ${ }^{6}$ Kushner and colleagues have found no difference between 7 and 5 courses of chemotherapy, 5 courses being adequate to achieve a response rate (RR) of $80 \%$ in children with high-risk neuroblastoma. ${ }^{7}$ The optimal duration of induction is still debated with the quality of response representing a major treatment-related prognostic factor. $^{2,3,8}$

Platinum-based agents, doxorubicin, epipodophyllotoxins, vincristine, ifosfamide and cyclophosphamide (CPM) have all been used in different combination treatments, thus representing the cornerstone of all multiagent induction chemotherapy approaches. ${ }^{2-9}$ Topotecan (TPT) has also shown antitumour activity against neuroblastoma in preclinical models ${ }^{10}$ and in the clinical setting. ${ }^{11} \mathrm{~A}$ combination of TPT and high-dose (HD) CPM has been reported to be highly effective in relapsed patients. ${ }^{12,13}$

In 2004, a pilot study was started by the Children Oncology Group (COG) to assess toxicity and feasibility of adding dose-intensive TPT and CPM to a multi-agent chemotherapy induction regimen in newly diagnosed high-risk neuroblastoma patients. ${ }^{14}$ This pilot study led to the current COG high-risk protocol (ANBL 0532).

In 2001, we opened a single-institution pilot study for patients with newly-diagnosed high-risk NB. The induction started with two cycles of HD-TPT/HD-CPM, followed by two courses including ifosfamide, carboplatin and etoposide (ICE). The ICE course was chosen on the basis of our personal experience and previously published results. ${ }^{15,16}$

We report the response and toxicity data of this 4-course based induction regimen.

\section{Patients and methods}

\subsection{Patients}

Thirty-five consecutive children over one year of age with either metastatic (33 patients) or localised MYCN-amplified tumour (2 patients) were classified as having high risk disease and were enroled in the study from April 2001 to August 2007. The protocol was approved by the institutional review board. Informed consent was obtained from parents. Patient characteristics are summarised in Table 1.

\subsection{Evaluation of disease at diagnosis}

Evaluation of primary tumour was carried out by computed tomography (CT) or magnetic resonance imaging (MRI). Metastatic spread was assessed by total body CT scan and
Table 1 - Patient characteristics at diagnosis.

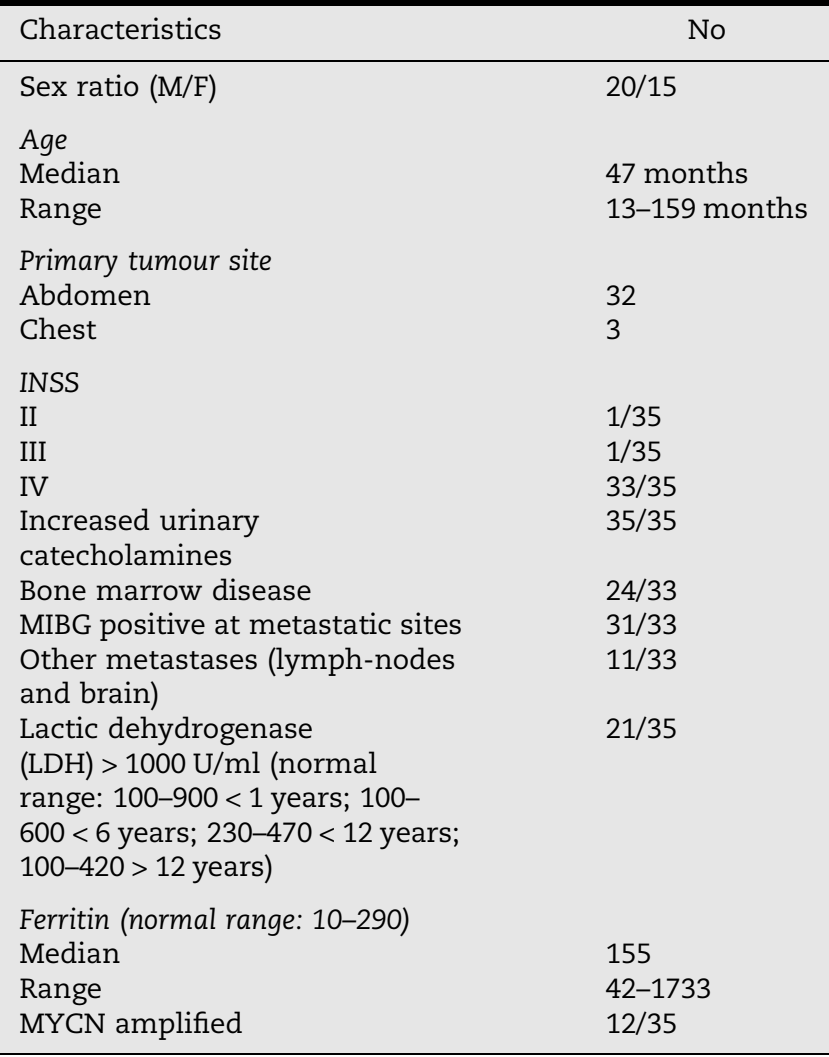

123-iodine-metaiodobenzylguanidine ( ${ }^{123}$ I-MIBG) scintigraphy and was completed by bilateral trephines and bone marrow aspirates performed for morphological and immunohistochemical evaluation. MYCN copy number was evaluated on tumour samples following current guidelines. ${ }^{17}$ Diagnosis and staging were performed according to the International Neuroblastoma Diagnosis and Staging Criteria. ${ }^{18}$

\subsection{Treatment}

The protocol included four courses of chemotherapy, repeated every 28 days or after haematopoietic recovery (defined as a neutrophil count $\geqslant 1000 / \mu \mathrm{L}$ and a platelet count $\geqslant 100,000 / \mu \mathrm{L}$ ) was achieved. Courses 1 and 2 consisted of HD-TPT and HD-CPM. Topotecan $\left(6 \mathrm{mg} / \mathrm{m}^{2}\right)$ was infused over $72 \mathrm{~h}$ and cyclophosphamide (140 mg/kg) over $48 \mathrm{~h}$, as previously reported. ${ }^{13}$ Courses 3 and 4 consisted of ICE: ifosfamide $\left(1800 \mathrm{mg} / \mathrm{m}^{2} /\right.$ day, total dose $\left.9 \mathrm{~g} / \mathrm{m}^{2}\right)$ was administered from day 1 to day 5 as a 3-h infusion; etoposide $\left(100 \mathrm{mg} / \mathrm{m}^{2} /\right.$ day, total dose $500 \mathrm{mg} / \mathrm{m}^{2}$ ) from day 1 to day 5 as a 2-h infusion; and carboplatin $\left(400 \mathrm{mg} / \mathrm{m}^{2} /\right.$ day, total dose $\left.800 \mathrm{mg} / \mathrm{m}^{2}\right)$ on days 1 and 2 as a 1-h infusion. Mesna was administered IV at the same daily dose of CPM or ifosfamide, preceded by a $600 \mathrm{mg} / \mathrm{m}^{2}$ bolus (ICE courses) for prevention of haemorrhagic cystitis.

Collection of peripheral blood stem cell (PBSC) for rescue after myeloablative therapy was planned after the third course or before if there was no evidence of bone marrow metastasis at diagnosis. 


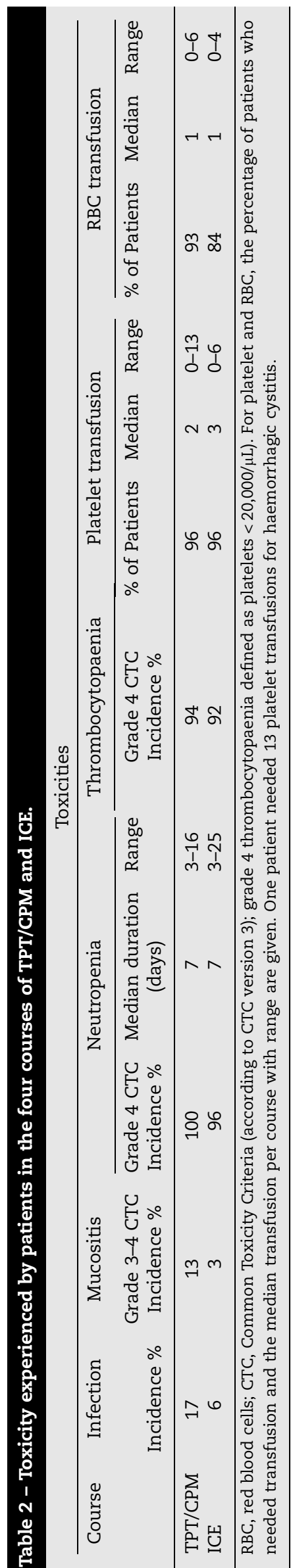

Surgical resection of primary tumour was routinely performed after the fourth course of chemotherapy. A single post-operative course of CAV followed surgery: this course included cyclophosphamide $\left(1500 \mathrm{mg} / \mathrm{m}^{2} /\right.$ day, total dose $3 \mathrm{~g} / \mathrm{m}^{2}$ ), which was administered on days 1 and 2 as a $3-\mathrm{h}$ infusion, doxorubicin $\left(25 \mathrm{mg} / \mathrm{m}^{2} /\right.$ day, total dose $\left.75 \mathrm{mg} / \mathrm{m}^{2}\right)$ and vincristine $\left(0.5 \mathrm{mg} / \mathrm{m}^{2} /\right.$ day, total dose $\left.1.5 \mathrm{mg} / \mathrm{m}^{2}\right)$ as a 72-h continuous infusion from day 1 to day 3 .

High-dose chemotherapy followed by peripheral blood stem cells (PBSC) rescue was performed in all patients achieving at least PR. The conditioning regimen consisted of either a combination of etoposide $\left(600 \mathrm{mg} / \mathrm{m}^{2}\right)$, thiotepa $\left(750 \mathrm{mg} / \mathrm{m}^{2}\right)$ and cyclophosphamide $(120 \mathrm{mg} / \mathrm{kg})$ or busulfan IV $(0.8-1.2 \mathrm{mg} / \mathrm{kg} /$ dose according to body weight for 16 consecutive doses over 4 days) plus melphalan (L-PAM, $140 \mathrm{mg} / \mathrm{m}^{2}$ in single dose). Hyperfractionated low-dose radiotherapy (21 Gy in two 1.5-Gy daily fractions, six hours apart, for seven consecutive days) was delivered to the primary tumour bed. ${ }^{19}$ Subsequently, 13-cis-retinoic acid was administered orally for 12 months at the standard dose of $160 \mathrm{mg} / \mathrm{m}^{2} /$ day for 14 consecutive days, and repeated every 28 days. $^{3}$

Granulocyte colony-stimulating factor (G-CSF; Filgrastim) $5 \mu \mathrm{g} / \mathrm{kg} \mathrm{IV} / \mathrm{SC}$ was started $48 \mathrm{~h}$ after each course of chemotherapy to accelerate neutrophil recovery. G-CSF was stopped when neutrophil count rebounded to $>500 / \mu \mathrm{L}$. Toxicity was evaluated in accordance with the International Common Toxicity Criteria version $3 .^{20}$

\subsection{Response evaluation}

Primary tumour response was evaluated after the second and fourth course, using the same investigations employed at diagnosis. The same radiologist (GN) reviewed all CT scans and MRI. Marrow evaluation was repeated after the second and fourth course, if positive at diagnosis. ${ }^{123}$ I-MIBG scan was repeated at the end of induction (after surgery and prior to high-dose treatment). All scans were reviewed by the same nuclear medicine physician (MCG). Responses were assessed according to the International Neuroblastoma Response Criteria. ${ }^{18} \mathrm{~A}$ major response (MR) was defined as partial, very good partial or complete response (PR/VGPR/CR).

\subsection{Statistical analysis}

Main aim of this pilot study was to improve the quality of response in newly high-risk neuroblastoma. End-points were major response rate and toxicity. A stopping rule based on the occurrence of toxic deaths was established. Descriptive analyses of response rate and toxicity were performed. The response rate is presented together with $95 \%$ confidence interval (CI).

Overall survival (OS) was defined as the time interval between the date of diagnosis and the date of death from any cause or the date of the last follow-up. EFS was defined as the time interval between the date of diagnosis and the date of first relapse/progression or the date of the last follow-up. The Kaplan-Meier method was used for estimating OS and EFS curves. ${ }^{21}$ Analysis was performed with the Stata 9.0 statistical software package (StatCorp LP, TX, USA). 


\section{Results}

\subsection{Treatment and toxicity}

All 35 patients enroled into the study were assessable for toxicity. Protocol violations occurred in four patients: in three cases surgery was performed after the fifth course in order to achieve a better local response and reduce surgical risks and, in one of them, CAV was replaced by a third ICE course; in one patient with congenital aortic stenosis CAV was replaced by TPT and carboplatin in order to avoid doxorubicin administration.

Dose reduction was required only once, namely in a patient developing the syndrome of inappropriate antidiuretic hormone secretion (SIADH).

The median length of the induction phase (from day 1 of the first course to day of surgery or to day 1 of the fifth course in patients who had undergone primary surgery) was 133 days (range 91-207 days). The median interval between courses was 30 days (mean value $30 \pm 4$ days, range 21-40 days); the median interval between TPT/CPM courses was 29 days (range 21-39 days), while for ICE courses it was 31.5 (range 24-40 days). The time interval between the first course and high-dose chemotherapy was 208 days (range 156-285). The median induction length for patients who did or did not achieve CR was 123 days (range 105139 days) and 136 days (range 91-208 days), respectively $(\mathrm{P}=\mathrm{NS})$.

Toxicity was mainly haematological with grade 4 neutropenia/thrombocytopenia and requirement of red blood cell transfusion in more than $90 \%$ of courses. Non-haematological toxicity was mainly gastrointestinal, with grade 3-4 mucositis in 11/140 (8\%) courses (see Table 2 for more details).

Neutropenic fever requiring hospitalisation occurred in 64/ 70 (91\%) HD-TPT/HD-CPM courses and in 40/70 (57\%) ICE courses, while blood stream infection/bacteremia was documented in 12/70 (17\%) HD-TPT/HD-CPM courses and in 4 (6\%) ICE courses. Neutropenic patients with fever were hospitalised and treated with intravenous antibiotics. No chemotherapy-related toxic death was reported during induction. One patient died after surgery for acute post-surgical renal impairment. No significant hearing loss was observed following chemotherapy. Peripheral blood stem cell (PBSC) collection was performed as intended but four patients required additional bone marrow (BM) harvesting due to an insufficient number of haematopoietic progenitors collected from peripheral blood.

\subsection{Response}

Primary tumour response was evaluated in 31 patients; 4 patients were already in CR after surgical removal of the primary tumour at time of diagnosis.

After the first two courses, the median percentage of tumour shrinkage was 56\% (range 0-90\%) with a mean of $57 \% \pm 23 \%$; this value reached $80 \%$ (range 0-97\%) with a mean of $74 \% \pm 22 \%$ after the fourth cycle.

Surgery was performed in 29 out of 31 patients: in 26 patients after four courses, while in 3 after the fifth course. Sixteen out of these 29 patients (55\%) achieved a CR after surgery: 15 out of 26 patients (58\%) who had surgery after four courses and 1 out of 3 patients after the fifth course.

Out of 33 patients with metastatic disease, 32 were evaluable for metastatic response. One child with PR at primary site and CR in bone marrow after the second course died of acute post-surgical complications, as mentioned above.

In terms of overall response, at the end of fifth course, 9 patients were in CR, 8 in VGPR and 12 in PR, with a major response rate of $85 \%$ (95\%CI 71-99\%). Forty-one percentage (95\% CI $23-57 \%$ ) of patients presented a complete remission at metastatic site and $34 \%$ (95\% CI $17-51 \%$ ), with metastatic spots on ${ }^{123}$ I-MIBG scan, achieved CR at the end of induction (see Table 3 for further details).

\subsection{Survival}

The median follow-up for the whole cohort of patients is 35 months (range 6-98 months). Of 35 patients, 24 (69\%) died. Relapse occurred in 27 (77\%) patients after a median of 14 months (range 6-37 months) from diagnosis. The 5-year OS and EFS were 31\% (95\% CI\% 16-47\%) and 20\% (95\% CI\% 9-35\%), respectively.

\section{Discussion}

During the last three decades several regimens have been employed as induction chemotherapy in high-risk neuroblastoma. ${ }^{2-9}$ TPT has shown antitumour activity against neuroblastoma cells both in preclinical models ${ }^{10}$ and in the clinical setting. ${ }^{11-13}$

Based on the promising results obtained with TPT and high-dose CPM in heavily pre-treated patients with neuroblastoma, ${ }^{13}$ we designed an induction regimen based on two HD-TPT/HD-CPM courses and two ICE courses, followed by surgery and one additional CAV course for the treatment of newly diagnosed high-risk neuroblastoma.

\begin{tabular}{|c|c|c|c|c|}
\hline & Tumour shrinkage & Bone marrow response & Major response rate & Response according to INRC \\
\hline After 2 courses & $57 \%+23 \%$ range $0-90 \%$ & $40 \%$ & $58 \%$ (95\%CI $41-75 \%)$ & \\
\hline After 4 courses & $74 \%+22 \%$ range $0-97 \%$ & $67 \%$ & $90 \%$ (95\%CI $80-100 \%)$ & \\
\hline Overall response & & $67 \%$ & $85 \%$ (95\%CI $71-99 \%)$ & CR $26 \%$ VGPR $24 \%$ PR $35 \%$ PD $15 \%$ \\
\hline
\end{tabular}

INRC, International Neuroblastoma Response Criteria; CR, complete response; VGPR, very good partial response; PR, partial response; PD, progressive disease; SD, stable disease. 
The aim of our study was to evaluate the feasibility and the impact of the proposed induction regimen in terms of major response rate prior to consolidation.

The proposed induction regimen resulted to be feasible with satisfactory dose intensity. Indeed, the median duration of induction was 133 days with a median time to high-dose chemotherapy of 208 days. According to our experience a shorter interval between courses seems possible; in particular, the interval between the HD-TPT/HD-CPM courses could be reduced to 21 days so as to start earlier the consolidation phase. Moreover, the post-operative course can be omitted considering the absence of any advantage in term of response after this course. As suggested by Pearson and colleagues, ${ }^{5}$ a rapid induction regimen enables to give much earlier a consolidation with high-dose chemotherapy, this potentially contributing to a better response and outcome. In order to compare our data with those previously reported, it is noteworthy that Pearson and colleagues reported a median time to consolidation of 176 days in the rapid treatment and of 231 days in the standard treatment. ${ }^{5}$

The toxicity of TPT/CPM and ICE was mainly haematological and this is consistent with previous reports on toxicity of TPT and other induction regimens. ${ }^{11-13,4,5,7}$ No toxic deaths were recorded.

The $85 \%$ major response rate at the end of induction is comparable with the results reported by North American studies such as CCG-3891, ${ }^{2}$ or by European studies., ${ }^{4,5}$ In the present study, a BM response was achieved in $42 \%$ of patients after 2 courses and in $67 \%$ after four/five courses, while a CR of metastatic sites was observed in $41 \%$ of patients, which is lower than that reported by Memorial Sloan-Kettering (MSK), ${ }^{22}$ but similar to that described in recent European studies. ${ }^{4,5}$ At MSK, the N6/N7 protocol achieved CR/VGPR of metastatic disease in $87 \%$ of treated children. ${ }^{7}$ A French Society of Paediatric Oncology (SFOP) study, however, failed to reproduce the results of the N6 protocol, and reported CR of metastatic sites in only $42 \%$ of patients, with CR in bone marrow in $82 \%{ }^{23}$ The response is comparable to the previous SFOP protocols NB87 and NB92, which were less toxic than the N6/N7 regimens. ${ }^{23}$ Pearson and colleagues ${ }^{5}$ similarly reported a CR percentage of less than $45 \%: 38 \%$ in the standard group and $44 \%$ in the rapid group (COJEC), although it should be underlined that the MIBG assessment was not mandatory in this study.

We recorded a major response rate of $58 \%$ at the primary tumour site after 2 HD-TPT/HD-CPM courses. Shortly after the start of this protocol, other authors reported a broad spectrum of major response rates in resistant or relapsed neuroblastoma treated with TPT as single agent or in combination with other drugs: Saylors and colleagues ${ }^{12}$ administered TPT $3.75 \mathrm{mg} / \mathrm{m}^{2}$ and CPM $1.25 \mathrm{~g} / \mathrm{m}^{2}$; Kushner and colleagues ${ }^{24}$ gave topotecan $6 \mathrm{mg} / \mathrm{m}^{2}$, vincristine $0.067 \mathrm{mg} / \mathrm{kg}$ and CPM $4.2 \mathrm{~g} / \mathrm{m}^{2}$; Garaventa and colleagues ${ }^{25}$ employed 2 courses of TPT $7.5 \mathrm{mg} / \mathrm{m}^{2}$, vincristine $2 \mathrm{mg} / \mathrm{m}^{2}$ and doxorubicin $45 \mathrm{mg} / \mathrm{m}^{2}$, reporting a major response rate between $18 \%$ and $56 \%$. These results are not directly comparable with our findings because they were obtained in a different patient population. The comparison seems more appropriate with three studies $^{26-28}$ in whom TPT was administered, either alone or in combination with other drugs, as an "up-front window" in newly diagnosed high-risk neuroblastoma. However, the comparison presents some limitations due to the partial evaluation of response in our series. Indeed, after two courses, we performed a CT scan of primary tumour, the urinary levels of vanillylmandelic and homovanillic acids and the bone marrow evaluation, but not the ${ }^{123}$ I-MIBG scan, which was repeated at the end of induction.

Our major response rate of $58 \%$ with a median tumour volume shrinkage of $56 \%$ and $\mathrm{BM}$ clearing in $42 \%$ matches that were reported recently by Santana and colleagues. ${ }^{26}$ In a pharmacokinetics-guided dose approach, 28 patients received a starting dose of TPT equal to $3 \mathrm{mg} / \mathrm{m}^{2}$ on the first day, followed by an adapted dose during the following 4 days repeated after 2 days of rest for 5 days every 28 days. The major response rate was $60 \%$, with a median tumour volume reduction of $58 \%$ : this protracted administration of TPT alone resulted in an impressive response. The overall pharmacokinetic targeting success rate was $72 \%$, with a median TPT dosage of $2.7 \mathrm{mg} / \mathrm{m}^{2}$ corresponding to an important cumulative dosage with a toxicity comparable to that observed in our study and a good dose intensity (a median of 57 days from start of cycle 1 to start of cycle 3). No toxic death was reported. The intersubject variability in TPT clearance was substantial. However, the individualised approach to dosing achieved the targeted systemic exposure with a limited and manageable toxicity.

Kretschmar and colleagues ${ }^{27}$ achieved a major response rate of $39 \%$ after 2 courses in 33 patients treated with TPT alone $\left(10 \mathrm{mg} / \mathrm{m}^{2}\right)$, and a major response rate of $29 \%$ in $34 \mathrm{pa}-$ tients treated with CPM $\left(1.25 \mathrm{~g} / \mathrm{m}^{2}\right)$ plus TPT $\left(3.75 \mathrm{mg} / \mathrm{m}^{2}\right)$. Simon and colleagues ${ }^{28}$ reported a major response rate of $72 \%$ with a combination of TPT $\left(1 \mathrm{mg} / \mathrm{m}^{2} /\right.$ day in a $168-\mathrm{h}$ continuous infusion, total dose $\left.7 \mathrm{mg} / \mathrm{m}^{2}\right)$, CPM $\left(100 \mathrm{mg} / \mathrm{m}^{2} /\right.$ day $1-\mathrm{h}$ infusion, days $1-7$, total dose $700 \mathrm{mg} / \mathrm{m}^{2}$ ) and etoposide $\left(100 \mathrm{mg} / \mathrm{m}^{2} /\right.$ day 1 -h infusion, days $\left.8-10\right)$; however, these impressive results were obtained in a limited population (11 patients) and need to be validated by the German Cooperative Group in their ongoing protocol for high-risk neuroblastoma.

As recently confirmed in a phase II study in relapsed neuroblastoma, TPT plus CPM was superior to TPT alone achieving a CR/PR in $39 \%$ of treated patients. ${ }^{29}$ In our experience the combination of these two drugs is feasible in newly diagnosed high-risk neuroblastoma, being able to reduce the tumour burden as confirmed by the median tumour shrinkage of $56 \%$ after two courses.

Although the proposed cumulative dosage of the two drugs is higher than that proposed in the COG studies, ${ }^{12,14}$ we did not observe any treatment-related death and toxicities were manageable. Indeed, only the conclusive data of the current COG high-risk neuroblastoma protocol will clarify the most effective dosage in term of response rate with lesser degree of toxicity.

Our TPT-based induction regimen achieved a major response rate comparable with the best results reported by the major national and international groups, with the notable exception of MSK. Toxicity was acceptable, with no toxic deaths occurring during induction. We suggest a TPT-based induction chemotherapy as an effective and well-tolerated option in newly diagnosed high-risk neuroblastoma. According to our experience, a shorter interval (21 days with adapted 
haematological limits such as neutrophil count $\geqslant 800 / \mu \mathrm{L}$ and a platelet count $\geqslant 75,000 / \mu \mathrm{L}$ ) between courses seems feasible both for HD-TPT/HD-CPM and for ICE courses in order to increase the dose intensity, to improve the quality of response and to give earlier myeloablation. Moreover, if included in future multicenter collaborative studies, a TPT pharmacokinetics-guided dosing approach and protracted administration, as proposed at St. Jude, ${ }^{26,30}$ could possibly further improve its efficacy.

\section{Conflict of interest statement}

None declared.

\section{Acknowledgements}

No writing assistance was utilised in the production of this manuscript. This study was partially presented at ASCO annual meetings 2008 and 2004 and SIOP annual meeting 2006. This study was partially supported by the Italian Ministry of Health (MADI) and the "Girasole Onlus" (MADI, II).

\section{R E F E R E N C E S}

1. Weinstein JL, Katzenstein HM, Cohn SL. Advances in the diagnosis and treatment of neuroblastoma. Oncologist 2003;8:278-92.

2. Matthay KK, Villablanca JG, Seeger RC, et al. Treatment of high-risk neuroblastoma with intensive chemotherapy, radiotherapy, autologous bone marrow transplantation, and 13-cis-retinoic acid. Children's Cancer Group. N Engl J Med 1999;341:1165-73.

3. Matthay KK, Reynolds CP, Seeger RC, et al. Long-term results for children with high-risk neuroblastoma treated on a randomized trial of myeloablative therapy followed by 13-cisretinoic acid: a children's oncology group study. J Clin Oncol 2009;27:1007-13.

4. De Bernardi B, Nicolas B, Boni L, et al. Disseminated neuroblastoma in children older than one year at diagnosis: comparable results with three consecutive high-dose protocols adopted by the Italian Co-Operative Group for Neuroblastoma. J Clin Oncol 2003;21:1592-601.

5. Pearson AD, Pinkerton CR, Lewis IJ, et al. High-dose rapid and standard induction chemotherapy for patients aged over 1 year with stage 4 neuroblastoma: a randomised trial. Lancet Oncol 2008;9:247-56.

6. Cheung NV, Heller G. Chemotherapy dose intensity correlates strongly with response, median survival and median progression-free survival in metastatic neuroblastoma. J Clin Oncol 1991;9:1050-8.

7. Kushner BH, Kramer K, LaQuaglia MP, et al. Reduction from seven to five cycles of intensive induction chemotherapy in children with high-risk neuroblastoma. J Clin Oncol 2004;22:4888-92.

8. Ladenstein R, Philip T, Lasset C, et al. Multivariate analysis of risk factors in stage 4 neuroblastoma patients over the age of one year treated with megatherapy and stem-cell transplantation: a report from the European Bone Marrow Transplantation Solid Tumor Registry. J Clin Oncol 1998;16:953-65.
9. Frappaz D, Michon J, Coze C, et al. LMCE3 treatment strategy: results in 99 consecutively diagnosed stage 4 neuroblastomas in children older than 1 year at diagnosis. J Clin Oncol 2000;18:468-76.

10. Zamboni WC, Stewart CF, Thompson J, et al. Relationship between topotecan systemic exposure and tumor response in human neuroblastoma xenografts. J Natl Cancer Inst 1998;90:505-11.

11. Blaney SM, Needle MN, Gillespie A, et al. Phase II trial of topotecan administered as 72-hour continuous infusion in children with refractory solid tumors: a collaborative Pediatric Branch, National Cancer Institute, and Children's Cancer Group Study. Clin Cancer Res 1998;4:357-60.

12. Saylors 3rd RL, Stine KC, Sullivan J, et al. Cyclophosphamide plus topotecan in children with recurrent or refractory solid tumors: a Pediatric Oncology Group phase II study. J Clin Oncol 2001;19:3463-9.

13. Kushner BH, Kramer K, Meyers PA, et al. Pilot study of topotecan and high-dose cyclophosphamide for resistant pediatric solid tumors. Med Pediatr Oncol 2000;35:468-74.

14. Park JR, Stewart CF, London WB, et al. A topotecan-containing induction regimen for treatment of high risk neuroblastoma.J Clin Oncol 2006;24(18S):9013 [ASCO Annual Meeting Proceedings Part I].

15. Donfrancesco A, Jenkner A, Castellano A, et al. Ifosfamide/ carboplatin/etoposide (ICE) as front-line, topotecan/ cyclophosphamide as second-line and oral temozolomide as third-line treatment for advanced neuroblastoma over one year of age. Acta Paediatr Suppl 2004;93:6-11.

16. Cairo MS, Shen V, Krailo MD, et al. Prospective randomized trial between two doses of granulocyte colony-stimulating factor after ifosfamide, carboplatin, and etoposide in children with recurrent or refractory solid tumors: a children's cancer group report. J Pediatr Hematol Oncol 2001;23(1):30-8.

17. Ambros IM, Benard J, Boavida M, et al. Quality assessment of genetic markers used for therapy stratification. J Clin Oncol 2003;21:2077-84.

18. Brodeur GM, Pritchard J, Berthold F, et al. Revisions in the international criteria for neuroblastoma diagnosis, staging and response to treatment. J Clin Oncol 1993;11:1466-77.

19. Kushner BH, Wolden S, LaQuaglia MP, et al. Hyperfractionated low-dose radiotherapy for high-risk neuroblastoma after intensive chemotherapy and surgery. $J$ Clin Oncol 2001;19:2821-8.

20. International Common Toxicity Criteria Version 3 (CTC.3). Available from URL: http://www.fda.gov/cder/cancer/ toxicityframe.htm.

21. Kaplan EL, Meier P. Nonparametric estimation from incomplete observation. J Am Stat Assoc 1959;53:457-81.

22. Cheung NK, Kushner BH, LaQuaglia M, et al. N7: a novel multi-modality therapy of high risk neuroblastoma (NB) in children diagnosed over 1 year of age. Med Pediatr Oncol 2001;36:227-30.

23. Valteau-Couanet D, Michon J, Boneu A, et al. Results of induction chemotherapy in children older than 1 year with a stage 4 neuroblastoma treated with the NB 97 French Society of Pediatric Oncology (SFOP) protocol. J Clin Oncol 2005;23:532-40.

24. Kushner BH, Kramer K, Modak S, et al. Camptothecin analogs (irinotecan or topotecan) plus high-dose cyclophosphamide as preparative regimens for antibodybased immunotherapy in resistant neuroblastoma. Clin Cancer Res 2004;10:84-7.

25. Garaventa A, Luksch R, Biasotti S, et al. A phase II study of topotecan with vincristine and doxorubicin in children with recurrent/refractory neuroblastoma. Cancer 2003;98:2488-94.

26. Santana VM, Furman WL, Billups CA, et al. Improved response in high-risk neuroblastoma with protracted 
topotecan administration using a pharmacokinetically guided dosing approach. J Clin Oncol 2005;20(23):4039-47.

27. Kretschmar CS, Kletzel M, Murray K, et al. Response to paclitaxel, topotecan, and topotecan-cyclophosphamide in children with untreated disseminated neuroblastoma treated in an upfront phase II investigational window: A Pediatric Oncology Group Study. J Clin Oncol 2004;22:4119-26.

28. Simon T, Längler A, Harnischmacher U, et al. Topotecan, cyclophosphamide, and etoposide (TCE) in the treatment of high-risk neuroblastoma. Results of a phase-II trial. J Cancer Res Clin Oncol 2007;133:653-61.
29. London WB, Frantz CN, Campbell LA, et al. Phase II randomized comparison of topotecan plus cyclophosphamide versus topotecan alone in children with recurrent or refractory neuroblastoma: a Children's Oncology Group study. J Clin Oncol 2010;28:3808-15.

30. Panetta JC, Schaiquevich P, Santana VM, et al. Using pharmacokinetic and pharmacodynamic modeling and simulation to evaluate importance of schedule in topotecan therapy for pediatric neuroblastoma. Clin Cancer Res 2008;14:318-25. 